14,15

\title{
Сдвиговой режим формирования градиента температуры в тонком нематическом канале
}

\author{
(C) А.В. Захаров ${ }^{1}$, С.В. Пасечник ${ }^{2}$, Д.В. Шмелева ${ }^{2}$ \\ ${ }^{1}$ Институт проблем машиноведения РАН, \\ Санкт-Петербург, Россия \\ ${ }^{2}$ Московский технологический университет, \\ Москва, Россия \\ E-mail: alexandre.zakharov@yahoo.ca, s-p-a-s-m@mail.ru, shmeliova@mail.ru
}

(Поступила в Редакцию 6 ноября 2018 г.

В окончательной редакции 6 ноября 2018 г.

Принята к публикации 7 ноября 2018 г.)

\begin{abstract}
Численными методами, в рамках нелинейного обобщения классической теории Эриксена-Лесли, с учетом баланса энтропии, сформулированы необходимые условия формирования градиента температуры $\nabla T$ в изначально однородно прогретом микроразмерном гибридно ориентированном жидкокристаллическом (ГОЖК)-канале под действием сдвигового напряжения $(\mathrm{CH})$. Рассмотрены случаи полной и частичной термической изоляции одной из поверхностей ГОЖК-канала при условии, что на другой поверхности поддерживается постоянная температура. Показано, как под действием СН, приложенного к одной из поверхностей этого ГОЖК-канала, можно разогреть планарно ориентированную границу канала и тем самым сформировать градиент температуры по сечению ЖК-канала.
\end{abstract}

Работа выполнена при финансовой поддержке РФФИ (грант № 16-02-00041a) и Минобрнауки (гранты 3.11888.2018/11.12 и 3.9585.2017/8.9).

DOI: 10.21883/FTT.2019.04.47430.305

\section{1. Введение}

Основу многих сенсоров и датчиков используемых в биотехнологических приложениях, медицине и фармокологии составляют термотропные и лиотропные жидкие кристаллы (ЖК) инкапсулированные в микро- и наноскопические объемы $[1,2]$. Один из способов позволяющий манипулировать такими микроразмерными системами основан на использовании внешнего электрического поля (электрокинетика) [2]. В основу другого способа позволяющего транспортировать микролитровые объемы ЖК-материалов, положено взаимодействие градиентов температуры и поля директора, которое ведет к формированию устойчивого гидродинамического потока v ЖК-материала [3]. Было показано, что необходимым и достаточным условием возникновения направленного горизонтального потока в гибридно-ориентированном ЖК (ГОЖК)-канале, в котором, например, ориентация поля директора на одной из ограничивающих поверхностей планарная, а на другой - гомеотропная, является взаимодействие градиентов температуры и поля директора. Ранее были сформулированы условия необходимые для начала транспортировки ЖК-капель инкапсулированных в микроразмерные каналы и капилляры под действием градиента температуры, создаваемого за счет разности температур на ограничивающих поверхностях [4]. Принимая во внимание тот факт, что такая разновидность ЖК-систем, как лиотропные ЖК, составляют основу многих сложных биологических организмов [5], то возможность манипулирования такими биосистемами посредством формирования градиентов температуры открывает новые перспективы в биотехнологических приложениях. Было также показано, что в изначально равномерно прогретом ГОЖК-канале под действием горизонтально направленного ЖК-потока с „треугольно-подобным“ профилем скорости $v_{\text {eq }}(z)$ по $z$-сечению ЖК-канала, характеризующимся ярко выраженным максимумом вблизи верхней планарно ориентированной поверхности, возможно формирование градиента температуры, направленного поперек микроразмерного ГОЖК-канала. Другими словами, таким направленным гидродинамическим потоком удалось разогреть верхнюю гомеотропно ориентированную ограничивающую поверхность и тем самым сформировать градиент температуры, направленный поперек ГОЖК-канала, микроскопической ширины.

В настоящей работе предлагается описание другого механизма, ответственного за формирование градиента температуры $\nabla T$ в ЖК-фазе под действием сдвигового напряжения $(\mathrm{CH})$ приложенного к одной из поверхностей ЖК-канала. Этот механизм, повидимому, может быть положен в основу наносенсоров, преобразующих механическую энергию в направленные градиенты температуры. В связи с этим возникает вопрос: при каких условиях возможно формирование $\nabla T$ в изначально однородно прогретом ЖК-канале под действием сдвигового напряжения приложенного к одной из поверхностей ЖК-канала.

Ответ на этот вопрос будет дан в рамках нелинейного обобщения классической теории Эриксена-Лесли $[6,7]$ 
с учетом баланса энтропии [8]. Будет рассмотрен такой температурный режим, когда температура на одной из ограничивающих поверхностях постоянна $\left(T_{\mathrm{lw}}=\mathrm{const}\right)$, a на другой ограничивающей поверхности допускается частичная или полная термическая изоляция. Таким образом, разность температур $\Delta T=T_{\text {up }}-T_{\mathrm{lw}}$, которая в начальный момент времени равна нулю, должна достичь максимально возможного значения, соответствующего существованию нематической фазы, для данной величины $\mathrm{CH}$ приложенного к одной из поверхностей ЖК-канала.

\section{2. Основные уравнения}

Рассмотрим гибридно-ориентированный ЖК-канал, в котором, например, ориентация поля директора на одной из ограничивающих поверхностей планарная $\left(\hat{\mathbf{n}}_{z=d} \| \hat{\mathbf{i}}\right)$, а на другой - гомеотропная $\left(\hat{\mathbf{n}}_{z=0} \| \hat{\mathbf{k}}\right)$. В нашем случае система координат отсчитывается от нижней ограничивающей поверхности ЖК-канала, где ось х и орт $\hat{\mathbf{i}}$ совпадают с направлением директора на верхней горизонтальной поверхности, в то время как ось $\mathrm{z}$ и орт $\hat{\mathbf{k}}$ направлены ортогонально ориентации директора на нижней ограничивающей поверхности ЖК-канала, а орт $\hat{\mathbf{j}}=\hat{\mathbf{k}} \times \hat{\mathbf{i}}$. Принимая во внимание тот факт, что ширина ЖК-канала $d \ll L$, где $L-$ длина канала, можно предположить, что все физические величины вовлеченные в процесс формирования градиента температуры $\nabla T$, зависят только от пространственной переменной $z$ и времени $t$. Таким образом, вектор поля директора $\hat{\mathbf{n}}=\left(n_{x}, 0, n_{z}\right)=n_{x} \hat{\mathbf{i}}+n_{z} \hat{\mathbf{k}}=\cos \theta(z, t) \hat{\mathbf{i}}+\sin \theta(z, t) \hat{\mathbf{k}}$ лежит в плоскости $\mathrm{xOz}$, причем $\theta(z, t)$ - полярный угол образованный директором $\hat{\mathbf{n}}$ и ортом $\hat{\mathbf{k}}$. Дальнейшее изложение будет дано для случая безразмерной переменной $\bar{z}=z / d$, причем верхняя черта над пространственной переменной в дальнейшем будет опущена.

В настоящей работе предлагается новый подход описывающий механизм формирования градиента температуры $\nabla T$ поперек ГОЖК-канала, под действием $\mathrm{CH} \sigma_{\mathrm{zx}}^{0}$ приложенного к верхней границе ЖК-канала

$$
\left(\sigma_{\mathrm{zx}}\right)_{\mathrm{z}=1}=\sigma_{\mathrm{zx}}^{0}
$$

когда на нижней ограничивающей поверхности поддерживается постоянная температура

$$
T_{\mathrm{z}=0}=T_{1},
$$

а на верхней ограничивающей поверхности допускается полная или частичная термическая изоляция

$$
-\lambda_{\perp}\left(T_{, z}\right)_{\mathrm{z}=1}=Q_{0} .
$$

Здесь $T_{, z}=\frac{\partial T}{\partial z}, \lambda_{\perp}-$ коэффициент теплопроводности ЖК-фазы в направлении перпендикулярном направлению директора $\hat{\mathbf{n}}$, а $Q_{0}$ - поток тепла через вехнюю границу ЖК-канала. Принимая во внимание тот факт, что толщина ЖК-канала варьируется в пределах нескольких микрометров, будем считать, что плотность ЖК-фазы постоянна по сечению канала $(\rho=\mathrm{const})$. Таким образом, мы имеем дело с несжимаемой ЖК-фазой, и условие несжимаемости принимает вид

$$
v_{\mathrm{x}, \mathrm{x}}(z, t)+v_{\mathrm{z}, \mathrm{z}}(z, t)=0,
$$

где $v_{x}$ и $v_{z}$ компоненты вектора скорости $\mathbf{v}(z, t)=v_{\mathrm{x}}(z, t) \hat{\mathbf{i}}+v_{\mathrm{z}}(z, t) \hat{\mathbf{k}}$. Последнее условие $\mathrm{c}$ учетом отсутствия проскальзывания на нижней границе ЖК-канала

$$
\left(v_{\mathrm{x}}(z, t)\right)_{\mathrm{z}=0}=0, \quad\left(v_{\mathrm{z}}(z, t)\right)_{\mathrm{z}=0}=0,
$$

приводит к тому, что в несжимаемой ГОЖК-канале существует только один гидродинамический поток, направленный параллельно ограничивающим поверхностям $\mathbf{v}(z, t)=v_{\mathrm{x}}(z, t) \hat{\mathbf{i}}=u(z, t) \hat{\mathbf{i}}$. В свою очередь, граничное условие для поля директора может быть записано в виде

$$
\theta_{\mathrm{z}=0}=0, \quad \theta_{\mathrm{z}=1}=\frac{\pi}{2} .
$$

Прежде всего следует отметить, что в отсутствии внешних полей устанавливается линейное распределение поля директора $\theta(z)=\frac{\pi}{2} z$ по сечению ЖК-канала. Под действием $\mathrm{CH} \sigma_{\mathrm{zx}}^{0}$ приложенного к верхней границе канала в ЖК-фазе устанавливаются балансы угловых моментов и импульсов действующих на единицу объема ЖК-фазы, а также баланс энтропии. Так баланс угловых моментов, записанный в безразмерной форме, принимает вид [3]

$$
\begin{aligned}
\gamma_{1}(\chi) \theta_{, \tau} & -\mathscr{A}(\theta) u_{, \mathrm{z}}-\left(\mathscr{G}(\theta) \theta_{, \mathrm{z}}\right)_{, \mathrm{z}}+\frac{1}{2} \mathscr{G}_{\theta}(\theta) \theta_{\mathrm{,z}}^{2} \\
& +\delta_{1} \theta_{, \mathrm{z}} \chi_{, \mathrm{z}}\left(\frac{1}{2}+\sin ^{2} \theta\right)=0,
\end{aligned}
$$

где $\chi(z, \tau)=T(z, \tau) / T_{\mathrm{NI}}-$ безразмерная температуpa, $\gamma_{1}=\frac{\bar{\gamma}}{\gamma_{10}}-$ безразмерный коэффициент вращательной вязкости, $T_{\mathrm{NI}}-$ значение температуры, соответствующее переходу нематик-изотропная жидкость, $\mathcal{A}(\theta)=\frac{\overline{\mathscr{A}}(\theta)}{K_{10}}$ и $\mathscr{G}(\theta)=\frac{\overline{\mathscr{G}}(\theta)}{K_{10}}-$ безразмерные функции полярного угла $\theta, \gamma_{10}$ и $K_{10}$ - максимальные значения коэффициентов $\gamma_{1}$ и $K_{1}$ в интервале температур, соответствующих нематической фазе, $\bar{A}(\theta)=\frac{1}{2}\left(\gamma_{1}-\gamma_{2} \cos 2 \theta\right)$, $\overline{\mathscr{G}}(\theta)=K_{1} \sin ^{2} \theta+K_{3} \cos ^{2} \theta, K_{1}$ и $K_{3}-$ коэффициенты упругости Франка, соответствующие поперечным и продольным деформациям, $\gamma_{i}(i=1,2)-$ коэффициенты вращательной вязкости, а $\tau=\frac{K_{10}}{\gamma_{10} d^{2}} \mid, t-$ безразмерное время. Здесь $\delta_{1}=\xi \frac{T_{\mathrm{NI}}}{K_{10}}-$ параметр ЖК-системы. Первые два члена в уравнении (7) описывают вклад вязкой силы, в то время как следующие два члена вклад упругой силы в баланс моментов действующих на единицу объема ЖК-фазы. Последний член представляет собой вклад термомеханической силы в баланс моментов [3]. Безразмерные уравнения баланса импульсов и энтропии имеют вид [3]

$$
\delta_{2} u_{, \tau}(z, \tau)=\sigma_{\mathrm{zx}, \mathrm{z}},
$$




$$
\begin{gathered}
\mathscr{P}_{, \mathrm{z}}(z, \tau)+\frac{\delta \mathscr{R}}{\delta \theta_{, \tau}} \theta_{, \mathrm{z}}=0, \\
\delta_{3} \chi_{, \tau}(z, \tau)=\left[\chi_{, z}\left(\lambda \cos ^{2} \theta+\sin ^{2} \theta\right)\right]_{, z} \\
+\delta_{4}\left[\chi \theta_{, \mathrm{z}}\left(\theta_{, \tau}\left(\frac{1}{2}+\sin ^{2} \theta\right)-\frac{3}{2} u_{, \mathrm{z}} \sin ^{2} \theta\right)\right] .
\end{gathered}
$$

Здесь $\quad \delta_{2}=\frac{\rho K_{10}}{\gamma_{10}^{2}}, \quad \delta_{3}=\frac{\rho C_{p} K_{10}}{\gamma_{10} \lambda_{\perp}} \quad$ и $\quad \delta_{4}=\xi \frac{K_{10}}{\gamma_{10} \lambda_{\perp} d^{2}} \quad-$ еще три параметра ЖК-системы, в то время как $\lambda=\lambda_{\|} / \lambda_{\perp}, C_{p}-$ коэффициент теплоемкости ЖК-фазы, а $\xi \sim 10^{-12}[\mathrm{~J} / \mathrm{m} \mathrm{K}]$ - термомеханическая постоянная [9]. Выражение для тангенциальной составляющей тензора напряжений $\sigma_{\mathrm{zx}}$ имеет вид

$$
\sigma_{\mathrm{zx}}=\frac{\delta \mathscr{R}}{\delta u_{, \mathrm{z}}}=h(\theta) u_{, \mathrm{z}}-\mathcal{A}(\theta) \theta_{, \tau}-\frac{3}{2} \delta_{1} \chi_{, \mathrm{z}} \theta_{\mathrm{z}} \sin ^{2} \theta,
$$

a $\mathscr{R}=\frac{\gamma_{10} d^{4}}{K_{10}^{2}} \overline{\mathscr{R}}-$ полная безразмерная диссипационная функция (ДФ) Релея $[3,6,7]$, которая имеет вид $\mathscr{R}=\mathscr{R}_{\text {vis }}+\mathscr{R}_{\text {tm }}+\mathscr{R}_{\text {th }}$, где $\mathscr{R}_{\text {vis }}=\frac{1}{2} h(\theta) u_{, \mathrm{z}}^{2}-\mathscr{A}(\theta) \theta_{, \tau} u_{, \mathrm{z}}$ $+\frac{1}{2} \gamma_{1} \theta_{, \tau}^{2}-$ вязкий, $\quad \mathscr{R}_{\mathrm{tm}}=\delta_{1} \chi_{, z} \theta_{, \tau} \theta_{, z}\left(\frac{1}{2}+\sin ^{2} \theta\right)$ $-\frac{3}{2} \delta_{1} \chi_{, \mathrm{z}} \theta_{\mathrm{z}} u_{\mathrm{z}, \mathrm{z}} \sin ^{2} \theta-$ термомеханический и $\mathscr{R}_{\mathrm{th}}=\frac{1}{2 \chi}$ $\times\left(\lambda \cos ^{2} \theta+\sin ^{2} \theta\right) \quad$ - термический вклады в ДФ Релея соответственно. В уравнении (9), $\mathscr{P}(z, \tau)=\frac{d^{2}}{K_{10}} P(z, \tau)$ - безразмерное гидростатическое давление в ЖК-канале, которое состоит из двух вкладов: вязкого вклада, определяемого величиной $\int_{0}^{z} \frac{\partial \mathscr{R}(z, \tau)}{\partial \theta_{, \tau}} \theta_{, z} d z$, и упругого вклада, определяемого потенциалом Франка $\frac{1}{2} \mathcal{G}(\theta) \theta_{\mathrm{z}}^{2}$.

Таким образом, формирование градиента температуры по сечению ГОЖК-канала, в котором верхняя граница полностью или частично термически изолирована и к которой приложено $\mathrm{CH} \sigma_{\mathrm{zx}}^{0}$, а на нижней поверхности поддерживается постоянная температура, описывается системой нелинейных дифференциальных уравнений в частных производных (7)-(10), и дополненных граничными условиями как для поля температуры

$$
\chi_{\mathrm{z}=0}=\chi_{1}, \quad\left(\chi_{, \mathrm{z}}\right)_{\mathrm{z}=1}=q_{0},
$$

так и поля директора

$$
\theta_{\mathrm{r}=0}=0, \quad \theta_{\mathrm{z}=1}=\frac{\pi}{2},
$$

где $q_{0}=-\frac{Q_{0} d}{T_{\mathrm{N} I} \lambda_{\perp}}-$ безразмерный тепловой поток через верхнюю границу ЖК-канала.

В случае ЖК-системы, образованной молекулами 4-n'-октил-n'-цианобифенила (8ЦБ), температурный интервал существования нематической фазы соответствует $307 \leq T \leq T_{\mathrm{NI}} \sim 313.8 \mathrm{~K}$. В этом интервале температур величины параметров $\delta_{1} \sim 24, \delta_{2} \sim 2 \cdot 10^{-6}$, $\delta_{3} \sim 6 \cdot 10^{-4}$, и $\delta_{4} \cdot 10^{-10}$ соответственно. Поэтому с учетом того, что $\delta_{i} \ll 1 \quad(i=2,3,4)$, уравнения Навье-Стокса (8) и теплопроводности (10) упрощаются. Так уравнения (8) и (10) принимают вид

$$
\begin{gathered}
h(\theta) u_{, \mathrm{z}}-\mathcal{A}(\theta) \theta_{, \tau}-\frac{3}{2} \delta_{1} \chi_{, \mathrm{z}} \theta_{\mathrm{z}} \sin ^{2} \theta=\sigma_{\mathrm{zx}}^{0}, \\
{\left[\chi_{, \mathrm{z}}\left(\lambda \cos ^{2} \theta+\sin ^{2} \theta\right)\right]_{, z}=0 .}
\end{gathered}
$$

Из уравнения (15) непосредственно следует, что градиент температуры, формирующийся по сечению ЖК-канала, пропорционален потоку тепла $q_{0}$ через верхнюю границу канала

$$
\chi_{, \mathrm{z}}(z)=\frac{q_{0}}{\lambda \cos ^{2} \theta+\sin ^{2} \theta} .
$$

Таким образом, из уравнения (16) следует, что в ГОЖК-канале с термически изолированной верхней границей градиент температуры $\chi_{z}(z)=0$, и вся механическая энергия $\mathrm{CH} \sigma_{\mathrm{zx}}^{0}$ диссипирует так, что в ЖК-канале генерируется гидродинамический поток $u(z, \tau)$, направленный параллельно ограничивающим поверхностям и вызывающий переориентацию поля директора $\theta(z, \tau)$. В случае частичной термической изоляции верхней границы ЖК-канала, подразумевающей наличие теплового потока $q_{0}$ внутрь либо из канала, по сечению ЖК-канала формируется градиент температуры, величина которого пропорциональна величине $q_{0}$, а его направление зависит от того, в какую сторону направлен тепловой поток.

\section{3. Решение гидродинамических уравнений и основные результаты}

Процесс переориентации поля директора $\hat{\mathbf{n}}(z, \tau)$, описываемый углом $\theta(z, \tau)$, перераспределение поля температуры $\chi(z, \tau)$ и формирование горизонтального потока $u(z, \tau)$ в ГОЖК-канале под действием $\mathrm{CH} \sigma_{\mathrm{zx}}^{0}$ приложенного к верхней границе канала описывается системой нелинейных дифференциальных уравнений в частных производных (14) и (16) совместно с граничными условиями (12)-(13) и начальными условиями для угла $\theta(\tau=0)=\frac{\pi}{2}$ и скорости $u(\tau=0)=0$ соответственно. Формирование разности температур по сечению ГОЖК-канала под действием $\mathrm{CH} \sigma_{\mathrm{zx}}^{0}$ приложенного к верхней границе было исследовано численно методом релаксаций [10], а критерием сходимости итерационной процедуры был выбран $\epsilon=\left|\left(\theta_{\mathrm{m}+1}-\theta_{\mathrm{m}}\right) / \theta_{\mathrm{m}}\right| \sim 10^{-4}$. Здесь $m-$ номер итерации. Как уже было отмечено выше, при отсутствии $\mathrm{CH} \sigma_{\mathrm{zx}}^{0}$ в ГОЖК-канале устанавливается линейное $\theta(z)=\frac{\pi}{2} z$ распределение поля директора, так, что на нижней границе $\theta_{\mathrm{z}=0}=0$ и директор соориентирован гомеотропно, в то время как на верхней границе $\theta_{z=1}=\frac{\pi}{2}$ и директор соорентирован планарно. Как только к верхней границе приложено $\mathrm{CH}$ $\sigma_{\mathrm{zx}}^{0}$, то происходит переориентация поля директора, и распределение угла $\theta(z, \tau)$ эволюционирует к его равновесному распределению $\theta_{\text {eq }}(z)$ по сечению ЖК-канала. 


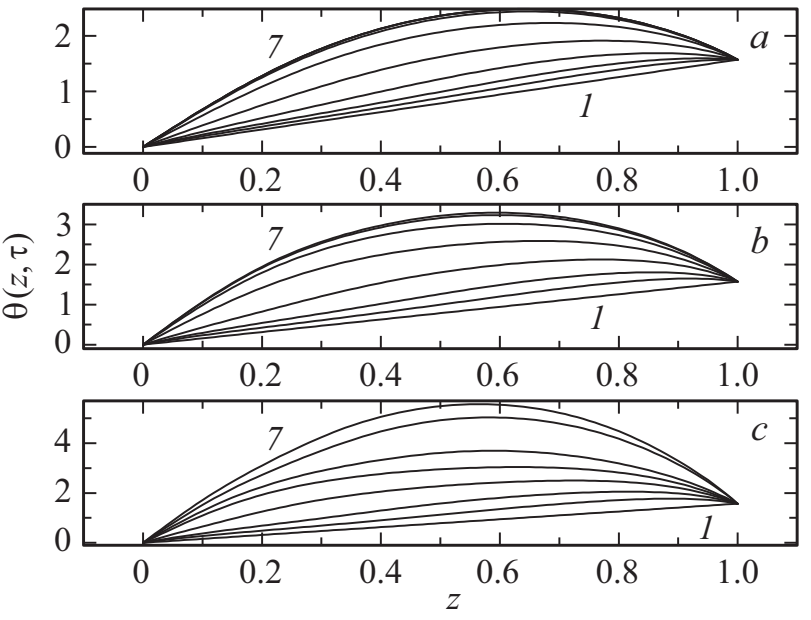

Рис. 1. Релаксация полярного угла $\theta(z, \tau)$ к равновесному распределению $\theta_{\mathrm{eq}}(z)$ по сечению ГОЖК-канала, соответствующему кривой 7, под действием $\mathrm{CH} \sigma_{\mathrm{zx}}^{0}=10(a), 20(b)$ и 30 (c) соответственно.

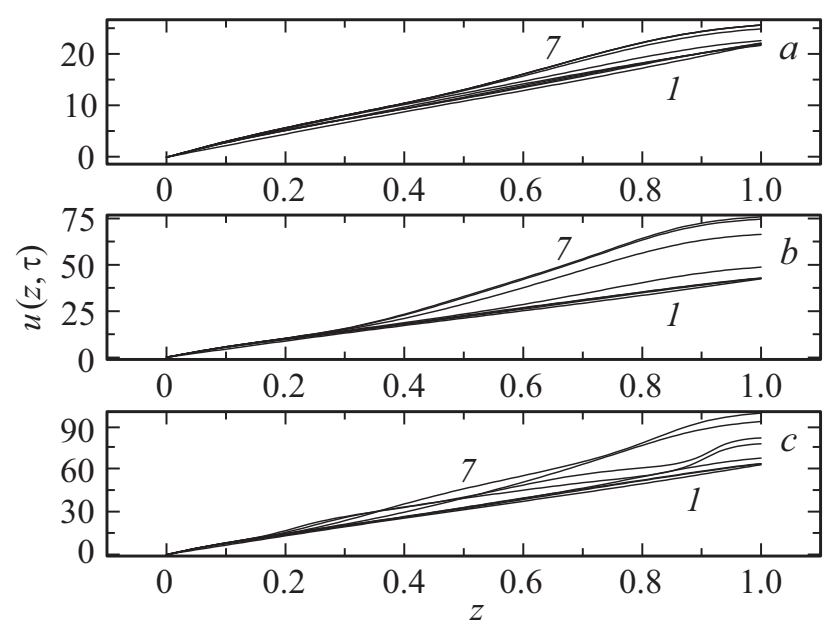

Рис. 2. Эволюция безразмерной скорости $u(z, \tau)$ к ее равновесному распределению $u_{\mathrm{eq}}(z)$ по сечению ГОЖК-канала, соответствующему кривой 7 , под действием $\mathrm{CH} \sigma_{\mathrm{zx}}^{0}=10(a)$, $20(b)$ и $30(c)$ соответственно.

На рис. 1 представлены результаты расчета эволюции полярного угла $\theta(z, \tau)$ к его равновесному распределению $\theta_{\text {eq }}(z)$ по сечению ГОЖК-канала для трех значений СН: $\sigma_{\mathrm{zx}}^{0}=10(\sim 5 \mathrm{~Pa})$ (случай I) (рис. $\left.1, a\right)$, $20(\sim 10 \mathrm{~Pa}) \quad$ (случай II) (рис. $1, b)$ и $30(\sim 15 \mathrm{~Pa})$ (случай III) (рис. 1,c) соответственно, причем величина теплового потока равна $q_{0}=0.02$, что соответствует величине размерного теплового потока $Q_{0}=200 \mathrm{~mW} / \mu \mathrm{m}^{2}$, направленного во внутрь через верхнюю границу ЖК-канала. Следует отметить, что величина размерного теплового потока $Q_{0}$ подобрана таким образом, чтобы вариации температуры соответствовали области существования нематической фазы. Во всех трех случаях (I, II, III) начальная ориентация поля директора характеризуется линейным распределением $\theta(z)=\frac{\pi}{2} z$ по сечению канала. Кривые $1-7$, рис. 1 представляют собой решения вышеописанной системы уравнений соответствующие следующим временам: $\tau_{1}=0.001$ (кривые 1$), \ldots \tau_{7}=\tau_{R}$ (кривые 7). Так в случае $\sigma_{\mathrm{zx}}^{0}=10$, $\tau_{R}=0.4 \quad(\sim 0.07 \mathrm{~s}), 20, \tau_{R}=0.32(\sim 0.055 \mathrm{~s})$ и 30 , $\tau_{R}=0.6(\sim 0.1 \mathrm{~s})$ соответственно. Согласно нашим расчетам, в случаях I, II, и III, в ГОЖК-канале формируется гидродинамический поток $u(z, \tau)$ направленный вдоль канала (см. рис. 2), причем максимальная величина $u_{\text {eq }}(\mathrm{III}) \sim 95(\sim 2.871 \mathrm{~mm} / \mathrm{s})$, практически в 4 раза больше максимального значения $u_{\text {eq }}(\mathrm{I}) \sim 25$ $(\sim 0.787 \mathrm{~mm} / \mathrm{s})$. Во всех этих трех случаях (I, II, III), под действием гидродинамического потока, обусловленного $\mathrm{CH} \sigma_{\mathrm{zx}}^{0}$, начальная ориентация поля директора претерпевает такое изменение, что поле директора в середине ГОЖК-канала закручивается практически на один оборот $\left(\theta_{\mathrm{eq}}(z=0.5)(\mathrm{III}) \sim 6\left(\sim 344^{\circ}\right)\right.$, относительно орта $\hat{\mathbf{j}}$. В случае такого теплового потока, как $Q_{0}=200 \mathrm{~mW} / \mu \mathrm{m}^{2}$, направленного во внутрь через верхнюю границу ЖК-канала, спустя время $t_{R}(\mathrm{I}) \sim 0.07 \mathrm{~s}$ $\left(\tau_{R}(\mathrm{I}) \sim 0.4\right)$ устанавливается равновесное распределение поля температуры по сечению ЖК-канала (кривая 7, рис. 3,a), характеризующееся тем, что на нижней границе $(z=0)$ поддерживается постоянная температура $T_{\mathrm{z}=0}=307 \mathrm{~K} \quad\left(\chi_{\mathrm{z}=0}^{\mathrm{eq}}=0.98\right), \quad$ в то время как на верхней границе устанавливается окончательная температура $T_{\mathrm{z}=1}^{\mathrm{eq}}=311.5 \mathrm{~K}\left(\chi_{\mathrm{z}=0}^{\mathrm{eq}}=0.995\right)$, для случая I, и $T_{\mathrm{z}=1}^{\mathrm{eq}}=311.3 \mathrm{~K}\left(\chi_{\mathrm{z}=0}^{\mathrm{eq}}=0.9946\right)$, для случая III соответственно. Таким образом, наибольшая разница $\Delta \chi=\chi_{\max }-\chi_{\min }=0.015(\sim 4.5 \mathrm{~K}$ в ГОЖК-канале формируется в случае І. Следует отметить, что с ростом величины $\mathrm{CH} \sigma_{\mathrm{zx}}^{0}$ с $10(\sim 5 \mathrm{~Pa})$ (случай I) до $30(\sim 15 \mathrm{~Pa})$ (случай III), максимальная величина угла $\theta$ возрастает с $\theta_{\max }(z \sim 0.6) \sim 3.0\left(\sim 172^{\circ}\right)$, в случае I, до $\theta_{\max }(z \sim 0.6) \sim 6.0\left(\sim 344^{\circ}\right)$, в случае III. Такое поведение угла $\theta$ физически обосновано, поскольку

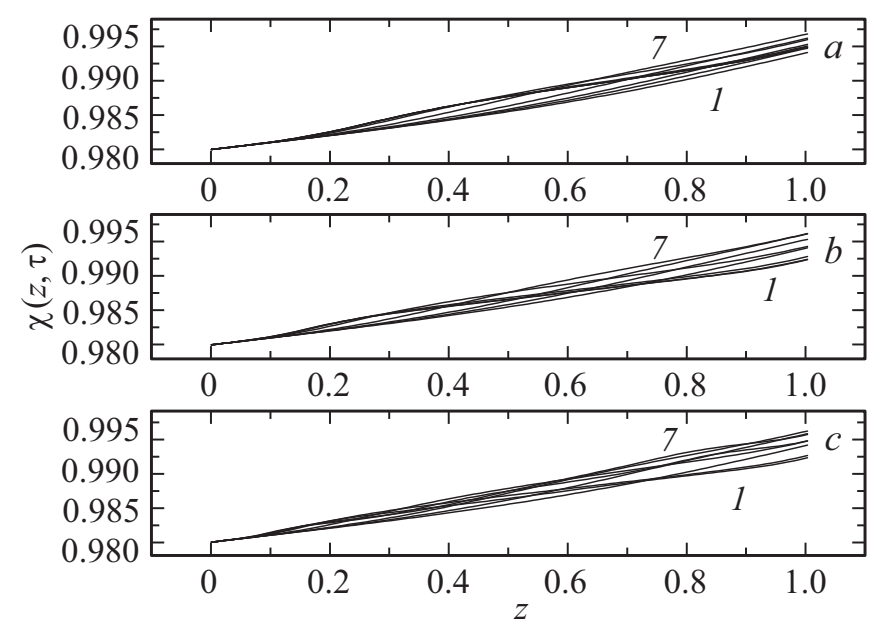

Рис. 3. Релаксация безразмерного поля температуры $\chi(z, \tau)$ к его равновесному распределению $\chi_{\text {eq }}(z)$ по сечению ГОЖК-канала, соответствующему кривой 7 , под действием $\mathrm{CH}$ $\sigma_{\mathrm{zx}}^{0}=10(a), 20(b)$ и $30(c)$ соответственно. 


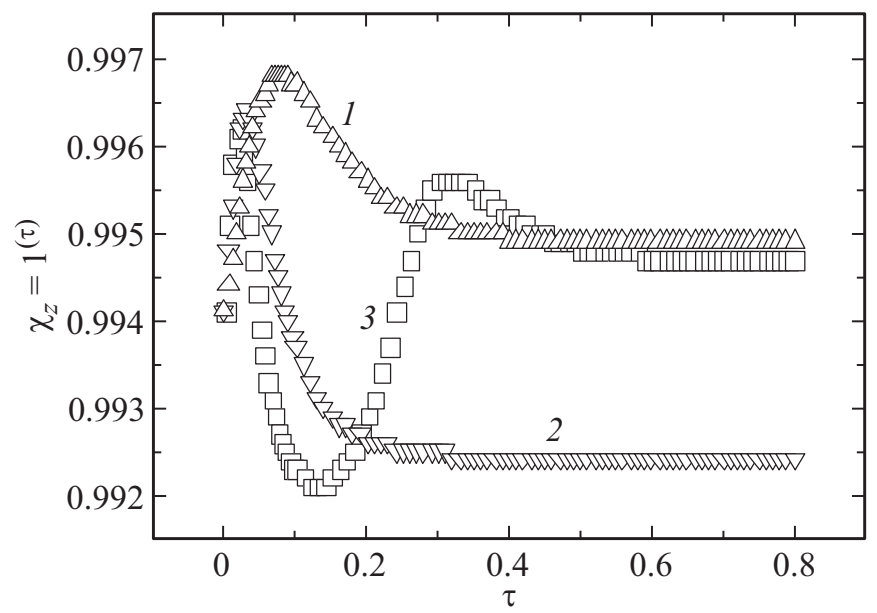

Рис. 4. Эволюция безразмерной температуры $\chi_{\mathrm{z}=1}(\tau)$ на верхней границе ГОЖК-канала под действием $\mathrm{CH} \sigma_{\mathrm{zx}}^{0}=10$ (кривая 1), 20 (кривая 2) и 30 (кривая 3) соответственно.

гидродинамическое течение сильнее отклоняет директор в середине ЖК-канала чем вблизи границ, где директор жестко соориентирован. В свою очередь, как только в ГОЖК-канале формируется гидродинамический поток $u(z, \tau)$ и происходит переориентация поля директора, по сечению канала начинает формироваться разность температур $\Delta \chi=\chi_{\max }-\chi_{\min }$. Здесь $\chi_{\min } \equiv \chi_{z=0}$. Результаты расчета эволюции $\chi_{\max }(\tau) \equiv \chi_{\mathrm{z}=1}(\tau)$ к равновесному значению $\chi_{\max }^{\mathrm{eq}}$, для трех величин $\mathrm{CH} \sigma_{\mathrm{zx}}^{0}$, с $10(\sim 5 \mathrm{~Pa})$ (случай I) до $30(\sim 15 \mathrm{~Pa})$ (случай III), представлены на рис. 4. Было установлено, что величина $\mathrm{CH} \sigma_{\mathrm{zx}}^{0}$ слабо влияет на величину равновесной максимальной температуры $\chi_{\max }^{\mathrm{eq}}$ формирующейся на верхней границе ЖК-канала.

Наши расчеты показали, что с изменением направления $\mathrm{CH}$ с положительного $\left(\sigma_{\mathrm{zx}}^{0}>0\right)$ на отрицательное $\left(\sigma_{\mathrm{zx}}^{0}<0\right)$ характер переориентации поля директора претерпевает как качественное, так и количественное изменение (см. рис. 5). В случае, когда СН $\sigma_{\mathrm{zx}}^{0}=-10(\sim-5 \mathrm{~Pa})$ (случай IV) (рис. 5, $\left.a\right)$, начальная ориентация поля директора, характеризующаяся линейной зависимостью полярного угла $\theta(z)=\frac{\pi}{2} z$ по сечению ГОЖК-канала, претерпевает такое изменение, что поле директора вблизи нижней границы канала переориентируется в отрицательном направлении, характеризующимся отрицательными значениями угла $\theta(z, \tau)$ (см. рис. $5, a)$. По мере переориентации поля директора, для случая I, минимальные значения полярного угла $\theta(z, \tau)$ достигают величины $\theta_{\text {eq }}(z \sim 0.4) \sim-0.6\left(\sim-34.4^{\circ}\right)$, а область отрицательных значений $\theta$ простирается до $z \sim 0.7$. С ростом величины $\mathrm{CH} \sigma_{\mathrm{zx}}^{0}<0$ с -10 до -30 (случай VI) (рис. 5,c), область отрицательных значений угла $\theta$ расширяется до $z \sim 0.9$ (случай VI (см. рис. 5, $c$ ), a минимальное значение полярного угла $\theta(z, \tau)$ достигают величины $\theta_{\text {eq }}(z \sim 0.5) \sim-3.0\left(\sim-172^{\circ}\right)$.

Такая сложная картина переориентации поля директора осуществляется под действием как гидродинамического потока, обусловленного $\mathrm{CH} \sigma_{\mathrm{zx}}^{0}<0$, так и термомеханической силы, обусловленной формирующимся градиентом температуры. Эволюция распределения поля скорости $u(z, \tau)$ по сечению ГОЖК-канала для трех величин $\mathrm{CH} \sigma_{\mathrm{zx}}^{0},-10$ (случай $\mathrm{IV}$ ), -20 (случай $\mathrm{V}$ ), и -30 (случай VI), представлена на рис. $6, a, b$ и $c$ соответственно. Времена релаксаций поля директора во всех трех случаях имеют следующие значения: в случае IV, $\tau_{R}=0.64(\sim 0.11 \mathrm{~s}), \mathrm{V}, \tau_{R}=0.47(\sim 0.08 \mathrm{~s})$ и VI, $\tau_{R}=0.48(\sim 0.08 \mathrm{~s})$ соответственно. Таким образом, наши расчеты показали, что генерируемое $\mathrm{CH}$ $\sigma_{\mathrm{zx}}^{0}$ поле скорости $u(z, \tau)$ оказывает сильное влияние на процесс переориентации поля директора. Так в случае VI, в центральной части ГОЖК-канала $(z \sim 0.5)$ ди-

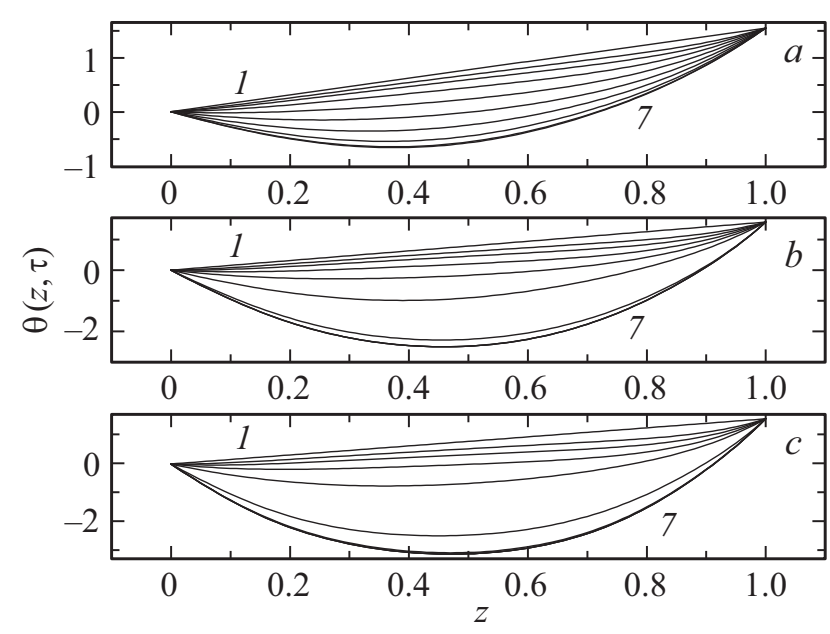

Рис. 5. Релаксация полярного угла $\theta(z, \tau)$ к равновесному распределению $\theta_{\mathrm{eq}}(z)$ по сечению ГОЖК-канала, соответствующему кривой 7 , под действием $\mathrm{CH} \sigma_{\mathrm{zx}}^{0}=-10(a),-20(b)$ и -30 (c) соответственно.

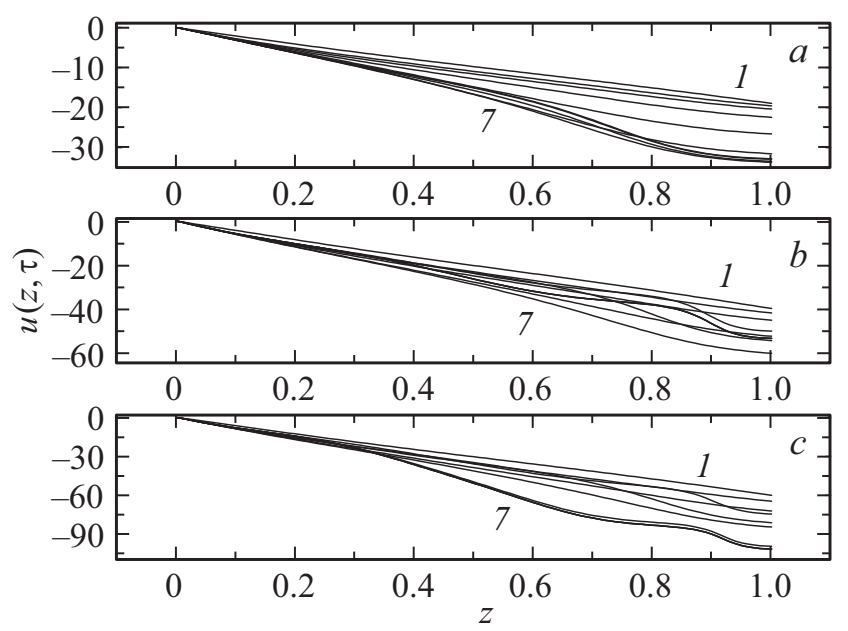

Рис. 6. Эволюция безразмерной скорости $u(z, \tau)$ к ее равновесному распределению $u_{\text {eq }}(z)$ по сечению ГОЖК-канала, соответствующему кривой 7 , под действием $\mathrm{CH} \sigma_{\mathrm{zx}}^{0}=-10(a)$, $-20(b)$ и $-30(c)$ соответственно. 


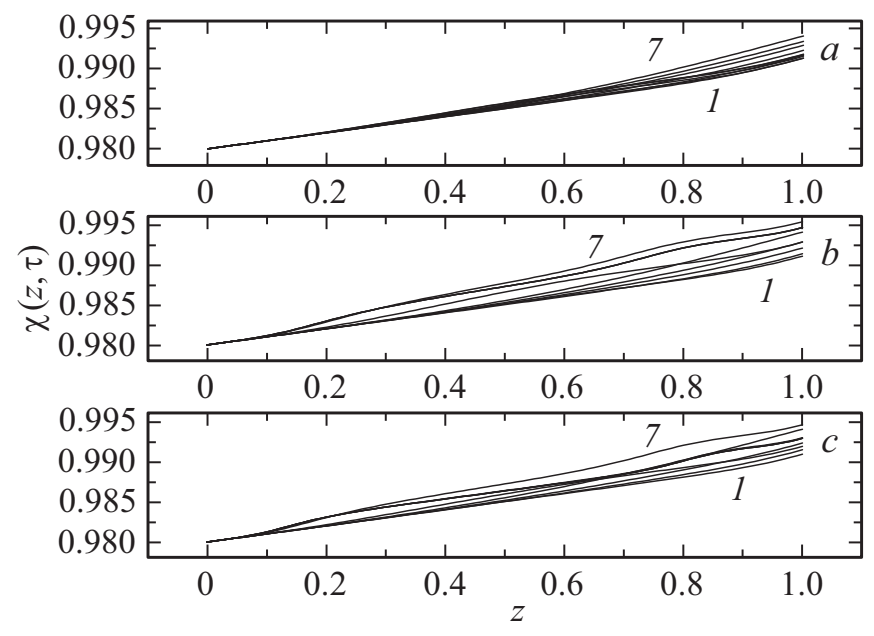

Рис. 7. Релаксация безразмерного поля температуры $\chi(z, \tau)$ к его равновесному распределению $\chi_{\mathrm{eq}}(z)$ по сечению ГОЖК-канала, соответствующему кривой 7 , под действием $\mathrm{CH}$ $\sigma_{\mathrm{zx}}^{0}=-10(a),-20(b)$ и $-30(c)$ соответственно.

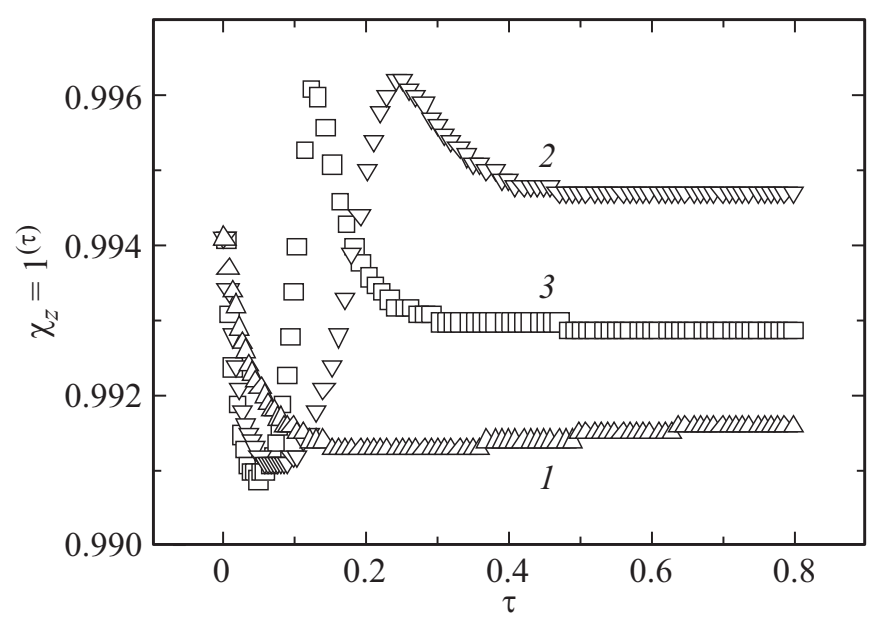

Рис. 8. Эволюция безразмерной температуры $\chi_{z=1}(\tau)$ на верхней границе ГОЖК-канала под действием $\mathrm{CH} \sigma_{\mathrm{zx}}^{0}=-10$ (кривая 1), -20 (кривая 2) и -30 (кривая 3) соответственно.

ректор соориентирован практически ортогонально границам ЖК-канала $\left(\theta_{\mathrm{eq}}(z \sim 0.5) \sim-3.0\left(\sim-172^{\circ}\right)\right.$ (см. рис. $5, c)$ ). Процесс релаксации поля скорости характеризуется монотонным ростом величины $|u(z, \tau)|$ по мере приближения к равновесному распределению $\left|u_{\mathrm{eq}}(z)\right|$ по сечению ГОЖК-канала. Максимальное абсолютное значение скорости $\left|u_{\mathrm{eq}}(z)=\frac{\gamma_{10} d}{K_{10}} v_{\mathrm{eq}}(z)\right|$, в случае IV, равно $33(1 \mathrm{~mm} / \mathrm{s})$, в случае $\mathrm{V}$, равно $60.4(1.86 \mathrm{~mm} / \mathrm{s})$ и в случае VI, равно $102(3.14 \mathrm{~mm} / \mathrm{s})$ соответственно. В случае отрицательного СН $\sigma_{\mathrm{zx}}^{0},-10$ (случай IV), -20 (случай $\mathrm{V})$, и -30 (случай $\mathrm{VI}$ ), на рис. $7, a, b$ и $c$, представлены эволюции поля температуры $\chi(z, \tau)$ к их равновесным распределениям $\chi_{\text {eq }}(z)$ по сечению ГОЖК-канала. В случае IV, мы имеем дело практически с линейной зависимостью $\chi(z, \tau)$ от пространственной переменной $z$, и слабой нелинейностью этой зависимости с ростом абсолютной величины $\left|\sigma_{\mathrm{zx}}^{0}\right|$. Для всех трех случаев IV, $\mathrm{V}$ и VI, равновесное распределение температуры по сечению ГОЖК-канала характеризуется тем, что на нижней границе $(z=0)$ поддерживается постоянная температура $T_{\mathrm{z}=0}=307 \mathrm{~K}\left(\chi_{\mathrm{z}=0}^{\mathrm{eq}}=0.98\right)$, в то время как на верхней границе устанавливается окончательная темпеpaтура $T_{\mathrm{z}=1}^{\mathrm{eq}}=310.4 \mathrm{~K}\left(\chi_{\mathrm{z}=0}^{\mathrm{eq}}=0.992\right)$ (см. рис. $\left.7, a\right)$, для случая IV, $T_{\mathrm{z}=1}^{\mathrm{eq}}=311.3 \mathrm{~K}\left(\chi_{\mathrm{z}=0}^{\mathrm{eq}}=0.9945\right)$ (см. рис. $\left.7, b\right)$, для случая $\mathrm{V}$ и $T_{\mathrm{z}=1}^{\mathrm{eq}}=310.8 \mathrm{~K} \quad\left(\chi_{\mathrm{z}=0}^{\mathrm{eq}}=0.993\right) \quad$ (см. рис. 7,c), для случая VI соответственно. Таким образом, наибольшая разница $\Delta \chi=\chi_{\max }-\chi_{\min }=0.0145$ $(\sim 4.35 \mathrm{~K})$ в ГОЖК-канале формируется в случае V. Результаты расчета эволюции $\chi_{\max }(\tau) \equiv \chi_{\mathrm{z}=1}(\tau)$ к равновесному значению $\chi_{\max }^{\mathrm{eq}}$, для трех величин $\mathrm{CH} \sigma_{\mathrm{zx}}^{0}$, с $-10(\sim-5 \mathrm{~Pa})$ (случай IV) до $-30(\sim-15 \mathrm{~Pa})$ (случай VI), представлены на рис. 8. Было установлено, что и в этом случае абсолютная величина $\mathrm{CH}\left|\sigma_{\mathrm{zx}}^{0}\right|$ слабо влияет на величину равновесной максимальной температуры $\chi_{\max }^{\mathrm{eq}}$ формирующейся на верхней границе ЖК-канала.

Следует отметить, что вышеописанные режимы формирования градиента температуры в ГОЖК-канале, под действием $\mathrm{CH} \sigma_{\mathrm{zx}}^{0}$ приложенного к верхней границе канала, при условии частичной термической изоляции верхней границы $\left(q_{0} \neq 0\right)$ и поддержании постоянной температуры на нижней границе $\left(T_{\mathrm{z}=0}=T_{1}\right)$ ЖК-канала, были описаны при условии отсутствия проскальзывания ЖК-материала на нижней границе канала (см. ур. (5)). В свою очередь, анализ поведения распределения скоростей $(u(z, \tau))$ по сечению ГОЖК-канала показал, что абсолютная величина скорости $\left|u(z, \tau)_{\mathrm{z}=1}\right|$ на верхней границе канала убывает с уменьшением абсолютной величины $\mathrm{CH}\left|\sigma_{\mathrm{zx}}^{0}\right|$ (см. рис. 2 и 6). Такая зависимость $u(z, \tau)_{\mathrm{z}=1}\left(\sigma_{\mathrm{zx}}^{0}\right)$ указывает на то, что при частичной термической изоляции верхней границы ЖК-канала, суще-

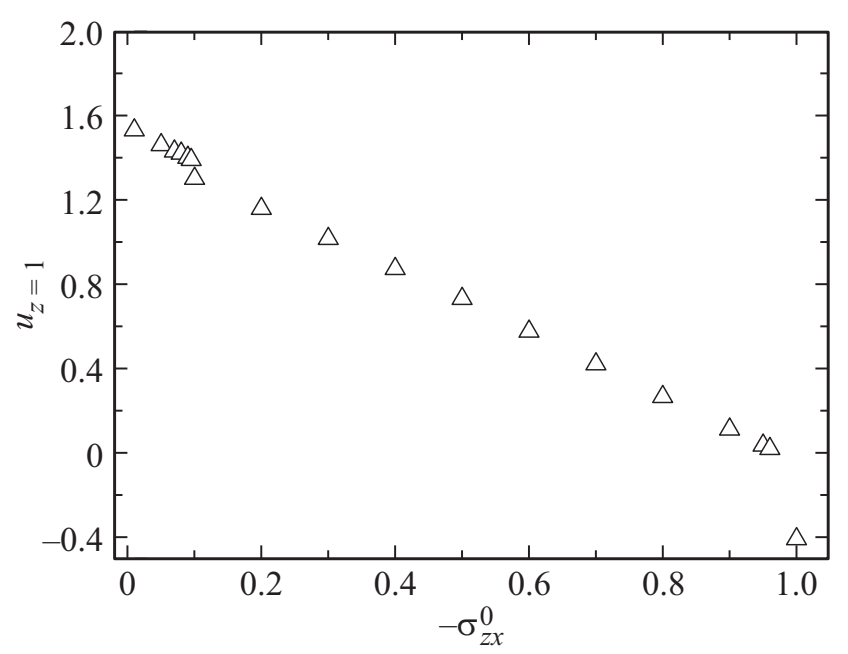

Рис. 9. Зависимость безразмерной гидродинамической скорости $u_{z=1}(z)$ на верхней границе ГОЖК-канала от величины СН $\sigma_{\mathrm{zx}}^{0}$ при фиксированном тепловом потоке $q_{0}=0.02$, направленным во внутрь ЖК-канала. 
ствует такое значение $\mathrm{CH} \sigma_{\mathrm{zx}}^{0}$ при котором отсутствует проскальзывание и на верхней границе ГОЖК-канала, т. е. $u_{\mathrm{z}=1}=0$. На рис. 9 представлены результаты расчета величины гидродинамической скорости на верхней границе ГОЖК-канала $u_{\mathrm{z}=1}$ в зависимости от приложенного $\mathrm{CH} \sigma_{\mathrm{zx}}^{0}$. Установлено, что с ростом абсолютной величины $\mathrm{CH} \sigma_{\mathrm{zx}}^{0}$ приложенного в отрицательном направлении к верхней границе ЖК-канала, величина скорости $u_{\mathrm{z}=1}$ убывает и вектор скорости $\mathbf{v}_{\mathrm{z}=1}=u(z, t)_{\mathrm{z}=1} \hat{\mathbf{i}}$ меняет направление при значениях $\sigma_{\mathrm{zx}}^{0} \sim-0.97(\sim-0.5 \mathrm{~Pa})$. При этом тепловой поток $q_{0}=0.02\left(Q_{0}=200 \mathrm{~mW} / \mu \mathrm{m}^{2}\right)$ был направлен во внутрь ЖК-канала.

\section{4. Заключение}

В предлагаемой работе численными методами, в рамках нелинейного обобщения классической теории Эриксена-Лесли, с учетом баланса энтропии, сформулированы необходимые условия формирование градиента температуры $\nabla T$ в изначально однородно прогретом микроразмерном гибридно-ориентированном жидкокристаллическом (ГОЖК)-канале под действием сдвигового напряжения $(\mathrm{CH})$ приложенного к одной из поверхностей этого канала. Рассмотрены случаи полной и частичной термической изоляции одной из поверхностей ГОЖК-канала при условии, что на другой поверхности поддерживается постоянная температура. Показано, как под действием $\mathrm{CH}$ можно разогреть планарно ориентированную границу ГОЖК-канала и тем самым сформировать градиент температуры, по сечению ЖК- канала. Величина $\nabla T$ пропорциональна величине теплового потока, направленного через верхнюю границу канала.

Следует отметить, что ранее было сформулировано условие формирования $\nabla T$ в ГОЖК-канале под действием стационарного, горизонтально направленного гидродинамического потока [11]. Было показано, что формирование $\nabla T$ возможно лишь в случае полной термической изоляции верхней гомеотропно ориентированной границы ГОЖК-канала, в то время как на нижней границе должна поддерживаться постоянная температура. Чиссленными методами было показано, что этот механизм формирования градиента температуры в ГОЖК-канале реализуется только лишь под действием гидродинамического потока со специфическим профилем скорости и при условии существования градиента поля директора.

В случае механической деформации под действием сдвигового напряжения, приложенного к планарно ориентированной границе ЖК-канала, по сечению канала градиент температуры $\nabla T$ формируется лишь при условии неполной термической изоляции этой границы. Величина этого $\nabla T$ пропорциональна величине теплового потока $q_{0}$, направленного через верхнюю ограничивающую поверхность. Если же достигалась полная термическая изоляция верхней ограничивающей поверхности при условии, что на нижней температура была постоянна, то вся механическая энергия, соответству- ющая сдвиговому напряжению, диссипировала в форме гидродинамического потока, направленного параллельно ограничивающим поверхностям ЖК-канала, вызывая таким образом переориентацию поля директора. Было показано, что изменение направления сдвигового напряжения (например, с положительного на отрицательное) качественно меняет характер релаксации поля директора. Все это свидетельствует о том, что характер сдвигового напряжения не только сильно влияет на ориентацию поля директора ЖК-канала, но и способен при определенных условиях термической изоляции ограничивающей поверхности сформировать градиент температуры по сечению ГОЖК-канала. Таким образом, мы надеемся, что настоящая работа дает ответы на некоторые вопросы, связанные с описанием релаксационных процессов, протекающих в ЖК-материалах под действием механической деформации, а также предлагает описание механизма, ответственного за возникновение градиента температуры в ЖК-каналах.

Исследованные в работе особенности, связанные с реакцией ЖК-материала инкапсулированного в тонкие и сверхтонкие каналы на локализованное воздействие сдвигового напряжения, необходимо учитывать при создании сенсоров и датчиков используемых в биотехнологических приложениях, медицине и биометрических оптических системах.

\section{Список литературы}

[1] R.B. Shoch, J.Y. Han, P. Renaud. Rev. Mod. Phys. 80, 839 (2008).

[2] W. Sparreboom, A. van den Berg, J.C.T. Eijkel. New. J. Phys. 12, 0115004 (2010).

[3] A.V. Zakharov, A.A. Vakulenko. J. Chem. Phys. 127, 084907 (2007).

[4] A.V. Zakharov, A.A. Vakulenko, M. Iwamoto. J. Chem. Phys. 132, 224906 (2010).

[5] S.J. Woltman, G.D. Jay, G.P. Crawford. Nature Mater. 6, 929 (2007).

[6] J.L. Ericksen. Arch. Ration. Mech. Anal. 4, 231 (1960).

[7] F.M. Leslie. Arch. Ration. Mech. Anal. 28, 265 (1968).

[8] С. Гроот, П. Мазур. Неравновесная термодинамика. Мир, M. (1964). $456 \mathrm{c}$.

[9] R.S. Akopyan, R.B. Alaverdian, E.A. Santrosian, Y.S. Chilingarian. J. Appl. Phys. 90, 3371 (2001).

[10] И.С. Березин, Н.Р. Жидков. Методы вычислений. Физматгиз, М. (1964). 464 с.

[11] A.V. Zakharov, A.A. Vakulenko. Chem. Phys. Lett. 454, 80 (2008).

Редактор Т.Н. Василевская 